\title{
Morphometrical development of tracheobronchial tree in human foetuses.
}

\author{
Işık Tuncer* \\ Department of Anatomy, Meram Faculty of Medicine, Necmettin Erbakan University, Konya, Turkey
}

\begin{abstract}
Objective: The purpose of this study was to investigate growth patterns in trachea, main bronchuses, lober bronchuses and thoracic cavity (TC) during the fetal period.

Method: This study was performed in Anatomy Department of Meram Faculty of Medicine Necmettin Erbakan University, and Dr. Faruk Sükan Maternity Hospital (Konya, Turkey). Thirty spontaneously aborted fetuses (18 males, 12 females) no malformations and variations were evaluated. The dimensions of the tracheobronchial tree (TBT) and TC were measured and the relationship between the data was statistically assessed with student's t-test.

Results: Means and standard deviations of the parameters according to gender and trimesters have been calculated. There was significant relation in all parameters between gestational age $(P<0.05)$. Right-left distance (TCtr) and superior-inferior distance (TCvr) was significantly higher in males to females. TCtr and TCvr changed with trimester.

Conclusion: Detailed information of TBT and TC related to the fetal period will reveal of biological variations of TBT and TC parameters to be used in future studies. We hope that data acquired in this study will facilitate other studies on TBT and TC anomalies, pathologies and variations as well as diagnoses and treatments of such as obstetrics, forensic medicine and fetal pathology.
\end{abstract}

Keywords: Trachea, Principal bronchus, Lober bronchus, TC, Fetal period, Development.

Accepted on September 03, 2018

\section{Introduction}

The endodermal respiratory primordium appears rounded and is generally known as the lung bud by 41 weeks the main bronchi are represented by right and left lung buds, which shortly are seen to arise namely, the trachea [1].

Congenital anomalies caused by external causes, such as genetic diseases, intrauterine infections and teratogenic agents in the early stages of gestation are important for prenatal diagnosis. Following fetal development consists of all parameters of the fetus. Fetal growth curves can be made for parametric assessments according to fetal age for all ethnic groups and may give us the normal standards of the fetal development of a population. Both invasive and non-invasive techniques are used for prenatal diagnosis. Fetal ultrasonography (USG), which is mostly used as a noninvasive technique, has the advantage of showing all of the fetal formations. To assess all stages of embryogenesis with USG, fetal morphometric anatomy should be known for every week of gestational age.

A study of over 200 standard postero-anterior (PA) chest Xrays showed the left main bronchus in 142 [2]. The mean diameter of the left main bronchus varied from 9 to $15.5 \mathrm{~mm}$. Although the mean diameter of the bronchi of females was less than of males', the mean bronchial diameter was not accurately predicted by the patient's weight or sex [3]. Only in males was bronchial diameter predicted by age and height.

Although many detailed studies in animals for the development of the TBT, there are not many studies in the human fetuses $[4,5]$. Especially after the development of imaging techniques, the morphometric data of the TBT is important for early diagnosis and treatments [6].

The knowledge of pathologies and anomalies of TBT and TC are important for early diagnosis and treatment. Detailed knowledge of TBT and TC development are not important in radiographic imaging. Morphometric data of fetal TBT and TC are either limited or belong to some specific weeks of gestational age. The reported reason of this is the difficulty to visualize and monitor the TBT and TC. Anatomical dissection method show more clear and reliable findings compared to radiological methods. In this study, our objective was to investigate the development of the TBT and TC of fetal collection from 15 to 40 weeks.

\section{Materials and Methods}

This study was carried out on 30 human fetuses (18 males, 12 females) between 15-40 weeks of gestation, in the years at 2017. Fetuses with no any external pathology or anomaly were obtained from the Gynecology Department of the Meram Faculty of Medicine of Necmetin Erbakan University, and Dr 
Faruk Sükan Maternity Hospital (Konya, Turkey) between 2015 and 2017 and were brought to the laboratory of Anatomy. Written consent from the families and an approval from the Ethics Board of the Meram Faculty of Medicine, Necmettin Erbakan University were obtained before the study (2008/172). Fetuses were assigned to three groups according to the gestational age as Group I ( $2^{\text {nd }}$ trimester, 12 fetuses), Group II ( $3^{\text {rd }}$ trimester, 11 fetuses) and Group III full term (term, 7 fetuses) comprised of fetuses with gestational ages between 15 and 25 weeks, between 26 and 37 weeks, and between 38 and 40 weeks.

Dissection was performed on $10 \%$ formaldehyde in the Anatomy Laboratory in Meram Faculty of Medicine, Necmettin Erbakan University. The thoracic wall was explored and TBT examined, they were controlled for any gross anomaly, and the location of the TBT and their relationships with neighbouring structures were determined. No pathologies were observed in the heart, oesophagus, lungs, larynx and they were all found to be in usual position.

In this study, a caliper, measuring tape, plastic ruler and compasses were used for the measurements of dimensions (Figure 1).

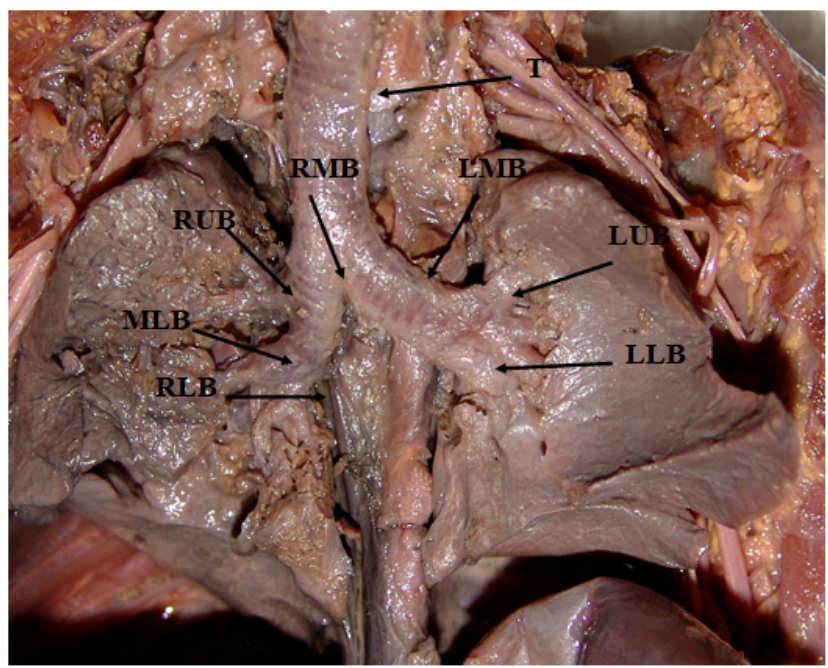

Figure 1. Anterior view of the TBT from one of the studied fetuses at 24 weeks of gestation after dissection and during performance of measurements. T: Trachea; RMB: Right Main Bronchus; LMB: Left Main Bronchus; RUB: Right Upper lobe Bronchus; MLB: Middle Lobe Bronchus; RLB: Right Lower lobe Bronchus; LUB: Left Upper lobe Bronchus; LLB: Left Lower lobe Bronchus.

Means of each parameter and standard deviations were computed with respect to gestational age and groups using SPSS statistical package (SPSS Inc., Chicago III., and USA). Non-parametric tests were used to compare groups due to the insufficient amount of data in some groups. The measured data were analyzed via Student's t-test (Statistical Package for Social Sciences Version 10.0 for Windows software) with respect to trimester. The difference between these groups was yielded a significant $\mathrm{P}$ value $(<0.05)$.

\section{Results}

The length (1), diameter (Ap: Anteroposterior; Tr: Transvers) and thickness (th) of the TBT (T: Trachea; RMB; Right Main Bronchus, RUB: Right Upper lobe Bronchus; MLB: Middle Lobe Bonchus; RLB: Right Lower lobe Bronchus; LMB: Left Main Bronchus; LUP: Left Upper lobe Bronchus; LLB: Left Lower lobe Bronchus) were measured. The means of these parameters were calculated for gender (Table 1). The size of the TBT were greater in males than females $(\mathrm{P}<0.05)$ (except for Tth, MLBap, LMB1, RLBth and LLBth) Means of the parameters with respect to gestational weeks are given in Table 2. There was a significant relation between all parameters for gestational age $(\mathrm{P}<0.001)$ Group comparisons showed a significant difference between all groups for all parameters $(\mathrm{P}<0.05)$. Trimester groups between which there were significant differences are presented (Table 2).

Table 1. Comparison of the data ( $m$ m, mean \pm standard deviation) of the TBT in human fetuses for gender.

\begin{tabular}{|c|c|c|}
\hline Parameters & Male (mean \pm SD) & Female (mean $\pm S D$ ) \\
\hline T-I & $24.85 \pm 9.69$ & $21.93 \pm 4.43$ \\
\hline T-ap & $3.25 \pm 0.72$ & $3.05 \pm 0.67$ \\
\hline T-tr & $5.56 \pm 1.60$ & $5.09 \pm 1.10$ \\
\hline T-th & $0.96 \pm 0.15$ & $0.96 \pm 0.07$ \\
\hline RMB-I & $4.59 \pm 1.53$ & $39.42 \pm 1.13$ \\
\hline RMB-ap & $4.57 \pm 1.52$ & $4.31 \pm 1.01$ \\
\hline RMB-tr & $3.08 \pm 0.94$ & $2.77 \pm 0.45$ \\
\hline RMB-th & $0.88 \pm 0.14$ & $0.82 \pm 0.16$ \\
\hline RUB-I & $22.29 \pm 0.69$ & $19.67 \pm 0.31$ \\
\hline RUB-ap & $2.65 \pm 0.78$ & $2.20 \pm 0.67$ \\
\hline RUB-tr & $2.16 \pm 0.65$ & $2.04 \pm 0.77$ \\
\hline RUB-th & $0.67 \pm 0.11$ & $0.65 \pm 0.09$ \\
\hline MLB-I & $45.53 \pm 1.91$ & $34.73 \pm 1.59$ \\
\hline MLB-ap & $1.93 \pm 0.65$ & $1.95 \pm 0.57$ \\
\hline MLB-tr & $1.82 \pm 0.61$ & $1.75 \pm 0.37$ \\
\hline MLB-th & $0.62 \pm 0.15$ & $0.57 \pm 0.06$ \\
\hline RLD-I & $60.82 \pm 2.51$ & $4.43 \pm 2.36$ \\
\hline RLB-ap & $3.11 \pm 1.12$ & $2.49 \pm 0.74$ \\
\hline RLB-tr & $2.03 \pm 0.60$ & $1.89 \pm 3.74$ \\
\hline RLB-th & $0.72 \pm 0.14$ & $0.72 \pm 0.16$ \\
\hline LMB-I & $4.00 \pm 3.08$ & $8.67 \pm 2.43$ \\
\hline LMB-ap & $4.42 \pm 1.12$ & $3.79 \pm 1.04$ \\
\hline LMB-tr & $2.67 \pm 0.58$ & $2.54 \pm 0.79$ \\
\hline LMB-th & $0.83 \pm 0.13$ & $0.78 \pm 0.11$ \\
\hline LUB-I & $26.88 \pm 0.77$ & $26.42 \pm 0.63$ \\
\hline
\end{tabular}




\begin{tabular}{lll}
\hline LUB-ap & $3.65 \pm 1.23$ & $2.86 \pm 0.83$ \\
\hline LUB-tr & $2.22 \pm 0.83$ & $1.78 \pm 0.56$ \\
\hline LUB-th & $0.70 \pm 0.17$ & $0.66 \pm 0.14$ \\
\hline LLB-I & $39.29 \pm 1.22$ & $32.75 \pm 0.83$ \\
\hline LLB-ap & $3.14 \pm 1.15$ & $2.47 \pm 0.60$ \\
\hline LLB-tr & $2.32 \pm 0.72$ & $2.05 \pm 0.54$ \\
\hline LLB-th & $0.68 \pm 0.4$ & $0.74 \pm 0.12$
\end{tabular}

\begin{tabular}{llll}
\hline RLB-tr & $1.71 \pm 0.41$ & $2.47 \pm 0.60$ & $1.97 \pm 0.61$ \\
\hline RLB-th & $0.68 \pm 0.13$ & $0.83 \pm 0.14$ & $0.73 \pm 0.15$ \\
\hline LMB-I & $82.39 \pm 2.12$ & $117.55 \pm 2.68$ & $95.72 \pm 2.88$ \\
\hline LMB-ap & $3.54 \pm 0.81$ & $5.17 \pm 0.75$ & $4.16 \pm 1.11$ \\
\hline LMB-tr & $2.28 \pm 0.48$ & $3.16 \pm 0.57$ & $2.62 \pm 0.66$ \\
\hline LMB-th & $0.75 \pm 0.09$ & $0.90 \pm 0.10$ & $3.81 \pm 0.12$ \\
\hline LUB-I & $22.94 \pm 0.51$ & $32.82 \pm 0.52$ & $26.69 \pm 0.70$ \\
\hline LUB-ap & $2.77 \pm 0.78$ & $4.23 \pm 1.05$ & $3.32 \pm 1.13$ \\
\hline LUB-tr & $1.67 \pm 0.49$ & $2.65 \pm 0.72$ & $2.04 \pm 0.75$ \\
\hline LUB-th & $0.63 \pm 0.14$ & $0.78 \pm 0.16$ & $0.69 \pm 0.16$ \\
\hline LLB-I & $34.22 \pm 0.68$ & $40.45 \pm 1.55$ & $36.59 \pm 1.11$ \\
\hline LLB-ap & $2.42 \pm 0.62$ & $3.60 \pm 1.12$ & $2.86 \pm 1.01$ \\
\hline LLB-tr & $1.88 \pm 0.49$ & $2.75 \pm 0.52$ & $2.21 \pm 0.66$ \\
\hline LLB-th & $0.66 \pm 3.13$ & $0.78 \pm 0.11$ & $0.70 \pm 3.13$ \\
\hline
\end{tabular}

Table 2. The mean parametric values $(\mathrm{mm}$, mean \pm standard deviation) about the TBT for trimesters.

\begin{tabular}{|c|c|c|c|}
\hline Parameters & Second trimester & Third trimester & Total \\
\hline T-I & $18.80 \pm 4.30$ & $32.11 \pm 5.53$ & $23.68 \pm 8.04$ \\
\hline T-ap & $2.85 \pm 0.52$ & $3.73 \pm 0.61$ & $3.17 \pm 0.70$ \\
\hline T-tr & $4.53 \pm 0.93$ & $6.82 \pm 0.82$ & $5.37 \pm 1.42$ \\
\hline T-th & $0.92 \pm 0.11$ & $1.03 \pm 0.11$ & $0.96 \pm 0.12$ \\
\hline RMB-I & $36.58 \pm 1.02$ & $55.00 \pm 1.21$ & $43.33 \pm 1.40$ \\
\hline RMB-tr & $2.55 \pm 0.46$ & $3.66 \pm 0.76$ & $2.96 \pm 0.79$ \\
\hline RMB-th & $0.80 \pm 0.11$ & $0.96 \pm 0.16$ & $0.86 \pm 0.15$ \\
\hline RUB-I & $18.05 \pm 0.34$ & $27.20 \pm 0.42$ & $21.21 \pm 0.57$ \\
\hline RUB-ap & $2.16 \pm 0.45$ & $3.00 \pm 0.91$ & $2.47 \pm 0.76$ \\
\hline RUB-tr & $1.81 \pm 0.37$ & $2.64 \pm 0.80$ & $2.11 \pm 0.69$ \\
\hline RUB-th & $0.62 \pm 0.08$ & $0.73 \pm 0.10$ & $0.66 \pm 0.10$ \\
\hline MLB-I & $34.89 \pm 1.41$ & $52.80 \pm 2.04$ & $41.29 \pm 1.84$ \\
\hline MLB-ap & $1.69 \pm 0.41$ & $2.42 \pm 0.67$ & $1.94 \pm 0.61$ \\
\hline MLB-tr & $1.56 \pm 0.36$ & $2.24 \pm 0.49$ & $1.79 \pm 0.52$ \\
\hline MLB-th & $0.56 \pm 0.10$ & $0.69 \pm 0.12$ & $0.60 \pm 0.12$ \\
\hline RLB-I & $47.33 \pm 2.25$ & $67.00 \pm 2.66$ & $54.36 \pm 2.54$ \\
\hline RLB-ap & $2.33 \pm 0.63$ & $3.85 \pm 0.87$ & $2.85 \pm 1.01$ \\
\hline
\end{tabular}

TCtr and TCvr were measured in each fetus. TCtr and TCvr showed sex difference $(\mathrm{P}<0.05)$ (Table 3$)$. TCtr and TCvr were significantly higher in males compared to females. TCtr showed laterality difference $(\mathrm{P}<0.05)$. TCtr was significantly higher on right side compared to left side. Means and standard deviations of these parameters for each gestational week and trimester were computed $(\mathrm{P}<0.05)$ (Table 4).

Table 3. Comparison of data obtained from dissected fetuses for lateralization (right-left) between genders (male-female) (mean $\pm S D$, $m m$ ).

\begin{tabular}{lllll}
\hline \multirow{2}{*}{ Parameters } & \multicolumn{3}{c}{ Right } & \multicolumn{3}{l}{ Left } \\
\cline { 2 - 5 } & Male & Female & Male & Female \\
\hline TCtr & $18.96 \pm 6.12$ & $16.61 \pm 3.80$ & $17.86 \pm 6.26$ & $15.92 \pm 3.98$ \\
\hline TCvr & $17.86 \pm 6.26$ & $15.92 \pm 3.98$ & $33.78 \pm 13.69$ & $33.63 \pm 7.77$
\end{tabular}

SD: Standard Deviation.

Table 4. Comparison of data obtained from dissected fetuses according to trimesters (second trimester-third trimester) and lateralization (rightleft) $($ mean $\pm S D, m m)$.

\begin{tabular}{lllll}
\hline \multirow{2}{*}{ Groups (weeks) } & Right & \multicolumn{2}{l}{ Left } \\
\cline { 2 - 5 } & TCtr & TCvr & TCtr & TCvr \\
\hline $2^{\text {nd }}$ trimester $(15-25$ weeks) & $14.95 \pm 2.34$ & $30.84 \pm 6.87$ & $14.17 \pm 2.29$ & $27.44 \pm 8.10$ \\
\hline $3^{\text {rd }}$ trimester (26-37 weeks) & $23.31 \pm 4.99$ & $42.75 \pm 5.37$ & $22.11 \pm 5.77$ & $44.56 \pm 7.91$ \\
\hline Total (15-40 weeks) & $18.02 \pm 5.36$ & $35.20 \pm 8.56$ & $17.09 \pm 5.47$ & $33.72 \pm 11.52$ \\
\hline
\end{tabular}

Standard deviation (SD).

\section{Discussion}

For the aim of conducting the current study, we obtained fresh autopsy specimens to ensure that the airway dimensions were close to dimensions in living patients. The fresh specimens allow preservations of physicochemical characteristics of the cartilaginous structures necessary to obtain similar physiologic conditions. In embedded specimens, because of tissue shrinkage, measurements were modified or impossible to 
realize (which corresponds to the abduction passive movement of the cricoarytenoid joint) [7].

Few studies have been published on the anatomical measurements of TBT in paediatric populations or premature populations.

In our study, the diameter and length of TBT were also measured, and their relationship with fetal age and other general parameters were determined. Fayoux [8] revealed the length parameters of trachea in fetuses aged between 15 and 41 weeks by micro-dissection and stated a linear increase. Mean length was 0.888 in theoretical age and 0.945 in corrected age respectively. In our study, there was also a correlation between gestational age and sizes of the TBT; a linear increase in TBT size was accompanying the gestational age (Table 2). When it is discussed with (Table 2) a more prominent increase was observed in the length of trachea than its diameter.

The results revealed about the length and diameter of trachea of Fayoux [8] is in consistent with our results; however, some differences are present in lower age groups (Table 2). As known, the trachea is a contractile organ [9]. Thus, differences in dimensions may be observed between live and post-mortem studies.

Chow et al. [10] found that the measurements of tracheal from a chest radiograph are less reliable in patients who are of small stature. They and others suggested CT as a possible alternative to measuring directly the diameters of the left main-stem bronchus [11-13]. This constitutes an exact and accurate gold standard method, but it is not always practical because in an emergency situation, accessible CT scans and experienced radiologists are necessary.

Since Jesseph et al. [14], who produced the first cadaveric about analysis of the dimensions of the TBT and several authors have described the dimension of the left main bronchus in cadavers [15], on chest radiographs [2,3], and on CT scans [11]. The measurements obtained from chest radiographs and CT scans are in the same range, except for reported by Chow et al. [11], which were obtained from an Asian population. They were roughly $15 \%$ less than those about cadaver investigation. This fact was outlined by Seymour [15], who found that the main tracheobronchial tree is essentially a passive structure for which the resting size is a function of the set of the incomplete cartilaginous rings, the pressure from surrounding structures, and the respiration (inspiration and expiration). Seymour [15] also demonstrated that the left main bronchus was nearly always of a uniform diameter. This observation made in cadavers was not confirmed in our study since our results showed that the left main bronchus is not circular. The left main bronchus is more frequently oval with a transverse-left main bronchus diameter greater than the anteroposterior-left main bronchus diameter.

In this study, we focused on the tracheobronchial development between $15^{\text {th }}$ and $40^{\text {th }}$ weeks of gestation and we investigated certain morphometric parameters. Owing to the anatomical dissections this is a pioneering work that provided detailed information that was not available before. It is thought that variations, anomalies or pathologies of the TBT seen during the puberty or adulthood may be connected with the intrauterine life. Better knowledge on developmental variations of the TBT will lead to better understanding of TBT variations and pathologies. Also our findings may be beneficial to those who intend to evaluate the development and the morphometry of the fetal TBT with obstetric US.

We hope that the data acquired in this study will contribute to the research carried by obstetricians, perinatologists, fetal pathologists and forensic medicine departments in determining developmental anomalies of the TBT, pathologies and variations during the fetal period.

\section{References}

1. Masters JRW. Epithelial-mesenchymal interaction during lung development: the effect of mesenchymal mass. Dev Biol 1976; 5: 98-108.

2. Hampton $\mathrm{T}$, Armtrong S, Russell WJ. Estimating the diameter of the left main bronchus. Anaesth Intensive Care 2000; 28: 540-542.

3. Hannallah MS, Benumof JL, Ruttiman E. The relationship between left main stem bronchial diameter and patient sizes. J Cardiothor Vasc Anesthesia 1995; 9: 119-121.

4. Weichselbaum M, Sparrow MP, Everett AW. Mapping the innervation of the bronchial tree in fetal and postnatal pig lung using antibodies to PGP 9.5 and SV2. Am J Respir Cell Mol Biol 1996; 15: 703-710.

5. Sparrow MP, Warwick SP, Everett AW. Innervation and function of the distal airways in the developing bronchial tree of fetal pig lung. Am J Respir Cell Mol Biol 1995; 13: $518-525$.

6. Deoras K, Wolfson M, Searls R, Hilfer R, Shaffer TH. Developmental changes in tracheal structure. Pediatr Res 1991; 30: 170-175.

7. Eckel HE, Koebke J, Sittel C. Morphology of the human larynx during the first five years of life studied on whole organ serial sections. Ann Otol Rhinol Laryngol 1999; 108: 232-237.

8. Fayoux P, Marciniak B, Devimse L, Storme L. Prenatal and early postnatal morphogenesis and growth of human laryngotracheal structures. J Anat 2008; 213: 86-92.

9. Yamaguchi T, Kohrogi H, Kawano O, Ando M, Araki S. Neutral endopeptidase inhibitör potentiates endothelin-1 induced airway smooth muscle contraction. J Appl Physiol 1992; 73: 1108-1113.

10. Chow MY, Liam BL, Thng CH, Chong BK. Predicting the sizes of the double-lumen endotracheal tube based on tracheal diameter. Anest Analg 1998; 87: 158-160.

11. Chow MY, Liam BL, Thng CH, Chong BK. Predicting the sizes of the double- lumen endotracheal tube using computed tomographic scan measurement of the left main bronchus diameter. Anesth Analg 1999; 88: 302-305.

12. Eberle B, Weiler N, Vogel N, Kauczor HU, Heinrichs W. Computed tomography-based tracheobronchial image reconstruction allows selection of the individually 
appropriate double- tube sizes. J Cardiothorac Vasc Anesth 1999; 13: 532-537.

13. Hanallah M, Benumof JL, Silverman PM, Kelly LC, Lea D. Evaluation of an approach to choosing a left doublelumen tube size based on chest computed tomographic scan measurement of left mainstem bronchial diameter. J Cardiothorac Vasc Anesth 1997; 11: 168-171.

14. Jesseph JE, Merendino KA. The dimensional interrelationships of the major components of the human tracheobronchial tree. Surg Gynecol Obstet 1957; 105-210.

15. Seymour AH. The relationship between the diameters of the adult cricoid ring and main tracheobronchial tree: a cadaver study to investigate the basis for double lumen tube selection. J Cardiothorac Vasc Anesth 2003; 17: 299-301.

\section{*Correspondence to}

Işı1k Tuncer

Department of Anatomy

Meram Faculty of Medicine

Necmettin Erbakan University

Konya

Turkey 